The Project of Theory.

Contribution to the study and precision of the theory of the architectural Project

PALABRAS CLAVE | TEORÍA-PRAXIS | PROYECTUALIDAD | POÉTICA | PROCESO CRÍTICO

KEYWORDS | THEORY-PRAXIS | PROJECTUALTTY | POETICS | CRITICAL PROCESS

\section{| RESUMEN |}

¿Pué es la Teoría del Proyecto? ¿Cómo opera? Y ¿Para qué es necesaria en el ejercicio arquitectónico? Son las preguntas que quisiera responder este estudio. El uso disperso y poco riguroso con que aparece en el discurso contemporáneo esta noción le confieren un velo de imprecisiones que imposibilita profundizar en sus contenidos y alcances. Frente a la inexistencia de una investigación argumental, comparativa y sistemática previa, se analizan y contrastan los contenidos de diez de los más importantes autores en la segunda mitad del siglo XX, concentrándose en la posibilidad de arribar a criterios que ayuden a comprender su potencial.

La aparente paradoja de originar conocimiento en un objeto aún no producido, cuya generación es a la vez su práctica y aprendizaje, es donde pueden encontrarse una serie de principios que lograrían establecerse como hipótesis o criterios atemporales transportables. Estrategias arquitectónicas que interesan al hacer, alcanzables por un proceso que posee en la elaboración práctica, crítica, poética y teórica sus instantes continuos.

\section{ABSTRACT}

What is Project Theory? How does it work? And Why is in necessary in the architectural exercise? These are questions that this investigation tries to answer.

The dispersed and sloppy use with which this notion appears in contemporary discourse, confer a veil of inaccuracies that precludes going deeper in their contents and scope. Facing the absence of a prior systematic investigation, arguments of the most important authors are analyzed and contrasted, focusing on the possiblity of reaching criteria thated, focusing on the possibility of reaching criteria that will help to understand their potential. The apparent paradox of originating knowledge in an object not yet produced, whose generation is both its practice and learning is where pricis prestice and learning, is where a series of principles might be found could be established as hypotheses or timeless and transportable criteria. Architectural strategies, which are of interest to the doers, achievable through a process that possess its continuous moments in practical, critical, poetic and theoretical elaboration.

\title{
El Proyecto de la Teoría. Contribución al estudio y precisión de la teoría del proyecto arquitectónico*
}

\author{
FABIÁN BARROS DI GIAMMARINO**. Universidad de Magallanes, Chile·fbd.arq@gmail.com \\ Fecha de recepción 05/abril/2015 - Fecha de aceptación 30/julio/2015
}

\section{INTRODUCCIÓN Y CONSIDERACIONES PREVIAS}

"Toda teoría es práctica".

G. W. Goethe

Este artículo intenta sistematizar los argumentos de un acotado (diez) y poliédrico grupo de autores, que sin tener noción de estar trabajando en el mismo objetivo, construyen un posible cuerpo de conocimientos en torno a la de Teoría del Proyecto, específicamente el grupo de autores a estudiar se sitúa en dos fragmentos temporales convergentes de la segunda mitad del siglo XX: su década inicial y su década final, que se extiende hacia comienzos del actual siglo.

Durante los años 60 parece asistirse a una ruptura epistémica, muchos autores ven en esta década un punto de arranque temporal, en particular el año 1968 es utilizado -entre otros- por Jencks (1980), Ockman (2000) y Hays (2000) quienes, apoyados en el mayo francés, reconocen una fisura epistemológica en la teoría de la arquitectura, que hasta los textos COMPLEJIDAD Y CONTRADICCIÓN EN LA ARQUITECTURA (Venturi, 1966) y LA ARQUITECTURA DE LA CIUDAD (Rossi, 1966) estaba muy ligada a una implicación directa sobre el cómo hacer proyectual, tanto así que Hays apunta que estos dos textos en la actualidad ya no podrían ser considerados con seguridad teoría de la arquitectura, pues desde ese momento esta ha sido argumentada, como dice George Teyssot, desde el arte, la filosofía, la comunicación y la información, alejándose y propiciando una distancia con la reflexión sobre la práctica proyectual. Distinguiendo inicialmente entre ambas teorías y siguiendo lo propuesto por Pina (2004) podemos establecer provisionalmente que una Teoría de la Arquitectura establece los fundamentos de la Teoría del Proyecto, esta última se enfocaría en la discusión de conceptos

El presente artículo surge de la investigación en curso para la tesis doctoral que desarrolla el autor en el programa de Doctorado en Proyectos Arquitectónicos Avanzados del Departamento de Proyectos Arquitectónicos de la ETSAMUPM como continuidad y profundización de la tesis leída el año 2012 para la obtención del grado de Magíster en Didáctica Proyectual en la Universidad del Bío-Bío.

** Arquitecto por la Universidad Finis Terrae (2006) y Magíster en Didáctica Proyectual por la Universidad del Bío-Bío (201 1). El año 2012 obtiene la beca CONICYT para doctorado, desde ese año reside en Madrid, España, donde es candidato a Doctor en Proyectos Arquitectónicos Avanzados por la Escuela Técnica Superior de Arquitectura de Madrid (ETSAM) y miembro del grupo oficial de investigación de crítica arquitectónica (ARKRIT) de la Universidad Politécnica de Madrid (UPM), España. Desde el año 2008 es académico e investigador en el Departamento de Arquitectura de la Universidad de Magallanes, en Chile, donde asume primero su coordinación académica y posteriormente su dirección. Paralelamente ha sido miembro del Comité Consultivo del Consejo Nacional de la Cultura y las Artes (CNCA), publicado diversos artículos y presentado ponencias en congresos internacionales. 
más concretos, como por ejemplo: contexto, estructura, función y construcción, pero desde un nivel de máxima abstracción. Utilizando los cuatro niveles de relación recíproca entre teoría y práctica que construye el pedagogo Gilles Ferry (1991), la Teoría del Proyecto debería ubicarse entre los dos niveles superiores: el más alto, o de, la Práctica-Teórica que intenta entender cómo funciona el sistema (proyecto) y sus actores, alcanzando un nivel científico; y el siguiente nivel, llamado "Praxiológico", que reflexiona sobre el por qué y qué hacer, sugiriendo al cómo hacer.

La reflexión arquitectónica en la segunda mitad del siglo XX, con la ascensión del posestructuralismo y la posmodernidad, contribuye a complicar y enmarañar la práctica arquitectónica hasta límites insospechados para la claridad lograda por la Modernidad. Solà-Morales nos dirá que el pensamiento contemporáneo, desde Foucault en adelante, parte del desorden en la realidad, de la multiplicidad y de las diferencias como datos iniciales y debido a esto es "difícil pensar en reconstruir la trama originaria" (Sola-Morales, 2003, p. 263). Por otra parte, Deleuze y Guattari (1997) pondrán de manifiesto la inexistencia de una plataforma desde la que sea posible construir una visión del mundo, para que vuelva Solà-Morales (1995) y proclame la desaparición de cualquier tipo de referencia absoluta que coordine o cierre los sistemas de conocimiento y valores, solo quedando la imposibilidad de tener una visión global de la realidad.

Este contexto convulso ha influido y confundido profundamente la práctica arquitectónica de las últimas décadas, haciendo que cotidianamente veamos cómo la acción y el proceso proyectual son permeables a influencias de otras aéreas de conocimiento, incrementándose las explicaciones de proyectos basados en argumentos provenientes de terminologías prestadas de otras disciplinas (generalmente científicas o literarias), mal interpretadas y someramente comprendidas por arquitectos que buscan alguna certidumbre en su quehacer
(Ábalos, 2005). Haciéndose patente, como indican Herreros (2012) y Mabardi (2012), la modificación de la preocupación de ciertos profesionales que comienzan a transformar su interés desde los resultados del hacer hacia una búsqueda por investigar las formas o estrategias de hacer en lo proyectual.

Este impulso por encontrar certidumbre se apoya en la aparición, desde la ciencia, de una crítica que comporta el propósito de denunciar las deshonestidades intelectuales sin importar su procedencia. El libro IMPOSTURAS INTELECTUALES (1997) de Sokal y Bricmont nos ayudará para explicar el síntoma de arbitrariedad general y disciplinar. Estos autores demuestran que si los textos posmodernistas "parecen incomprensibles, es por la sencilla razón que no quieren decir nada" (1997, p. 23) y buscan alertar a la filosofía, las humanidades y las ciencias sociales sobre casos manifiestos de "charlatanería":

“Es particularmente sospechoso que conceptos matemáticos abstrusos, usados raramente en física -y ciertamente nunca en química o biología- se vuelvan milagrosamente pertinentes en las humanidades y las ciencias sociales" (Sokal \& Bricmont, 1997, p. 27).

Estos científicos se dedican minuciosamente a mostrar las incoherencias y errores de lógica en la articulación de las ideas y sus relaciones. Abogan por un pensamiento claro y lógico, pero del mismo modo que interesa aquello, demuestran interés por revelar el cómo; es decir: ¿̇Cuál es la secuencia de decisiones y principios que llevan a relacionar determinados elementos en una producción intelectual? Algo que en las ciencias, a diferencia de las disciplinas proyectuales, es requisito básico de validez.

Este contexto de ambigüedad intelectual es transportado a la arquitectura con exquisita fluidez, algunos casos emblemáticos son los textos de Tschumi, Koolhaas o Ito. Quizá el más radical sea EL FIN DE LO CLÁSICO DE EISENMAN (1984) donde promulga el fin de tres ficciones, siendo la más drástica de sus conclusiones, el fin de la verdad. Por contrapartida, este contexto de arbitrariedad, es denunciado por autores más académicos como Miranda (1999 y 2008), Montaner (1999) y Leach (1999) entre otros, y pareciera sintetizarlos José Antonio Marina al escribir:

"El talante posmoderno tiene un atractivo aire de ligereza, de juego, de falta de compromiso, de gusto por la incoherencia, que a todos nos parece refrigerante [...] En comparación con tanta frescura, las duras nociones de verdad, imperativo, voluntad, tan caras a la modernidad, aparecen como tarascas hostiles y amenazadoras" (Marina, 2004, p. 58).

Para este estudio es de particular interés el diagnóstico elaborado por Javier Seguí en su texto ESCRITOS PARA UNA INTRODUCCIÓN AL PROYECTO ARQUITECTÓNICO (1996) cuando detalla, luego de años de investigación, los factores que impiden teorizar sobre el proyecto a los arquitectos:

- La pastosidad e imprecisión del discurso sobre lo que se hace al proyectar, cuando salvo excepciones los autores no diferenciarían bien lo que son puras operaciones de los significados, proyecciones y deseos que las propias operaciones arrestan.

- La costumbre de los arquitectos de eludir la cita referencial, evitándola o negando su evidencia.

- El común empleo, sin excesivo rigor, de términos genéricos que se acaban distanciando de sus significados estrictos hasta desnaturalizarse en razón de significados diversos e implícitos.

- El relato de los proyectos consiste en justificaciones de la formalidad alcanzada en cada caso, donde coinciden los profesionales docentes y los no docentes.

- El no dar importancia a la aclaración de los procesos de proyecto.

- El rechazo de plano, sin aceptar ninguna posibilidad, de teorizar o metodizar el proyecto y el quehacer del que proyecta arquitectura. 
Frente a esta situación, advertida por Tafuri años antes como una "crisis de las creencias, de las instituciones y de las ideologías" (1968, p. 48), el autor afirma que el arquitecto participa sin lograr configurar autónomamente afirmaciones e instituciones nuevas. Reconoce que esto ha llevado a un aumento de iniciativas orientadas a la creación de una verdad individual. Esto es coincidente con lo descrito por Piñón cuando advierte del extremo relativismo de la expresión personal, que reconoce como una especie de "eufemismo con el que se quiere atenuar la patología común de quien actúa sin otro criterio que el relativismo insaciable del mercado y las limitaciones de su propio estado de ánimo" (2006, p. 136). Tafuri se esfuerza en argumentar que si bien al arte no le corresponde replegarse sobre sí mismo para poner de manifiesto el propio porqué, es el contexto relativista y posmo el que obliga, permite y compromete a ello.

Con este contexto disciplinar, este artículo se enfoca, más que en aquellos autores que presentan indicios de elaborar una particular Teoría del Proyecto, en quienes intentan aclarar los fundamentos epistémico-conceptuales de su constitución.

Desde ese ángulo de indagación no se pretende adquirir un carácter de relato histórico, como el elaborado por Gregotti en EL TERRITORIO DE LA ARQUITECTURA (1972), tampoco se intentan agotar las referencias sobre el tema, concentrándonos en los más importantes argumentos elaborados para precisar tres preguntas ineludibles pero, también, inalcanzables: ¿̇qué es la Teoría del Proyecto?, ¿̇cómo opera y actúa? y żpara qué es necesaria en el ejercicio arquitectónico?

Los objetivos concretos que persigue este estudio pueden ser sintetizados en cuatro pasos secuenciales: 1) identificar o reconocer los autores con aportes argumentales a la noción, 2) describir sus argumentos, 3) confrontar y relacionar estos argumentos en busca de afinidades o diferencias, y 4) explicitar y sistematizar los resultados de este cruce argumental.

1. Conjunto, necesariamente incompleto, de autores en relación a la Teoría del Proyecto, destacándose los que aportan fundamentos epistemológicos.

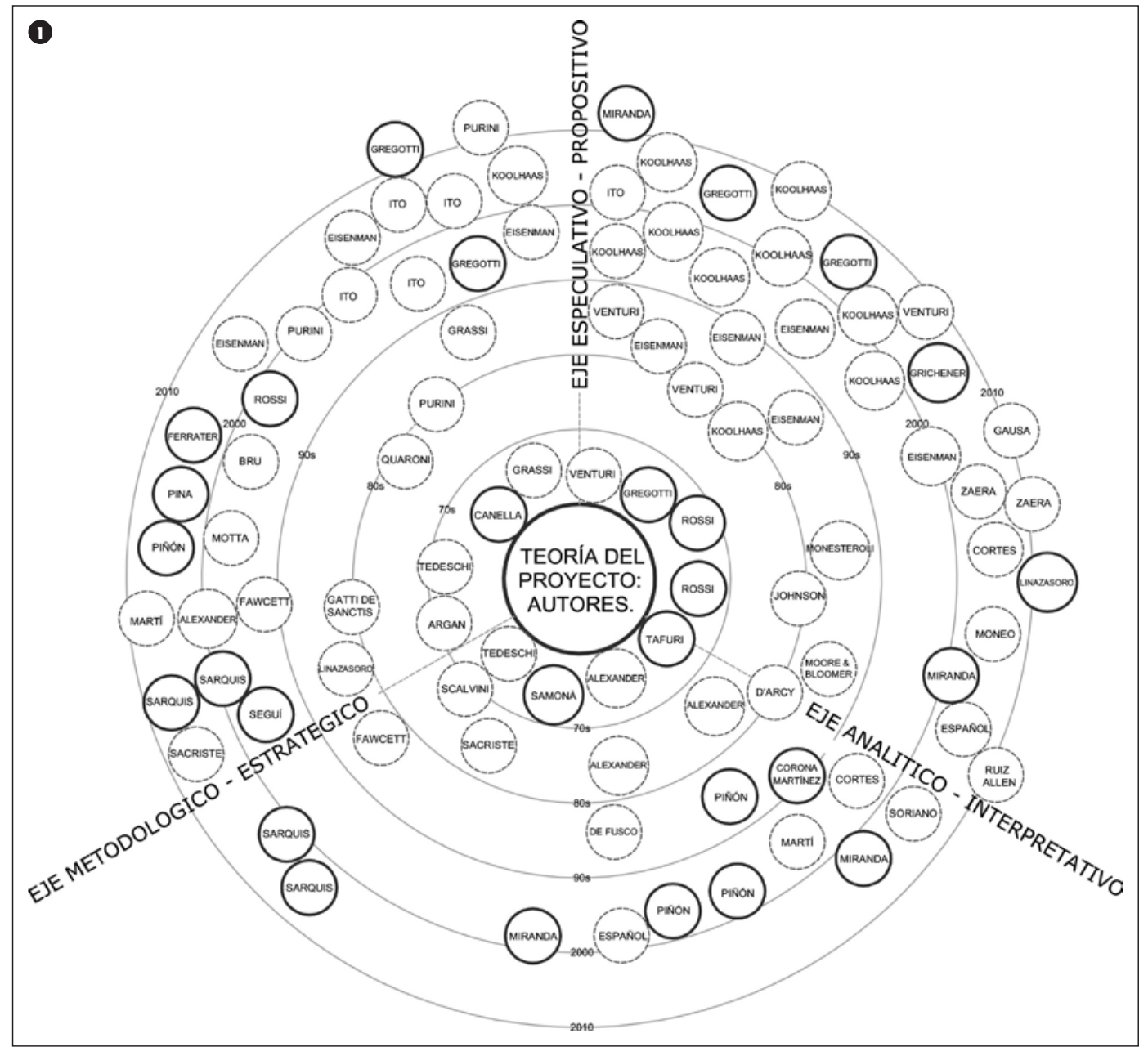

Para intentar conseguir estos objetivos, el estudio se planteó como teórico-"fenomenológico", descriptivo y correlacional. La metodología general consideró poner bajo escrutinio todas las posiciones, de modo de explorar la posibilidad de extraer por comparación un discurso coherente.

Para dar cumplimiento a lo anterior, y una vez obtenidas las fuentes bibliográficas primarias, es decir, los textos seleccionados en su versión original y completa de los autores que traten el tema, se procedió a su estudio de modo sistemático, esta actividad tuvo como método fundamental la descomposición y análisis del discurso de cada autor, extrayendo, definiendo y categorizando las ideas-constantes en su contenido. Considerando lo amplio e impreciso de las comprensiones y usos actuales de esta noción, se decidió trabajar -primeramente-en los contenidos de cada autor por separado para posteriormente producir su discusión y elaboración conjunta. De acuerdo a esto, se resuelve no tomar postura por ninguna formulación ni orientación determinada, a fin de no influenciar o dirigir la búsqueda. De aquí que el método empleado sea "fenoménico".

La entrada neutral a cada texto, junto con la postergación de juicios parciales, permitió 
2. Ejemplo de tabla comparativa formulada.

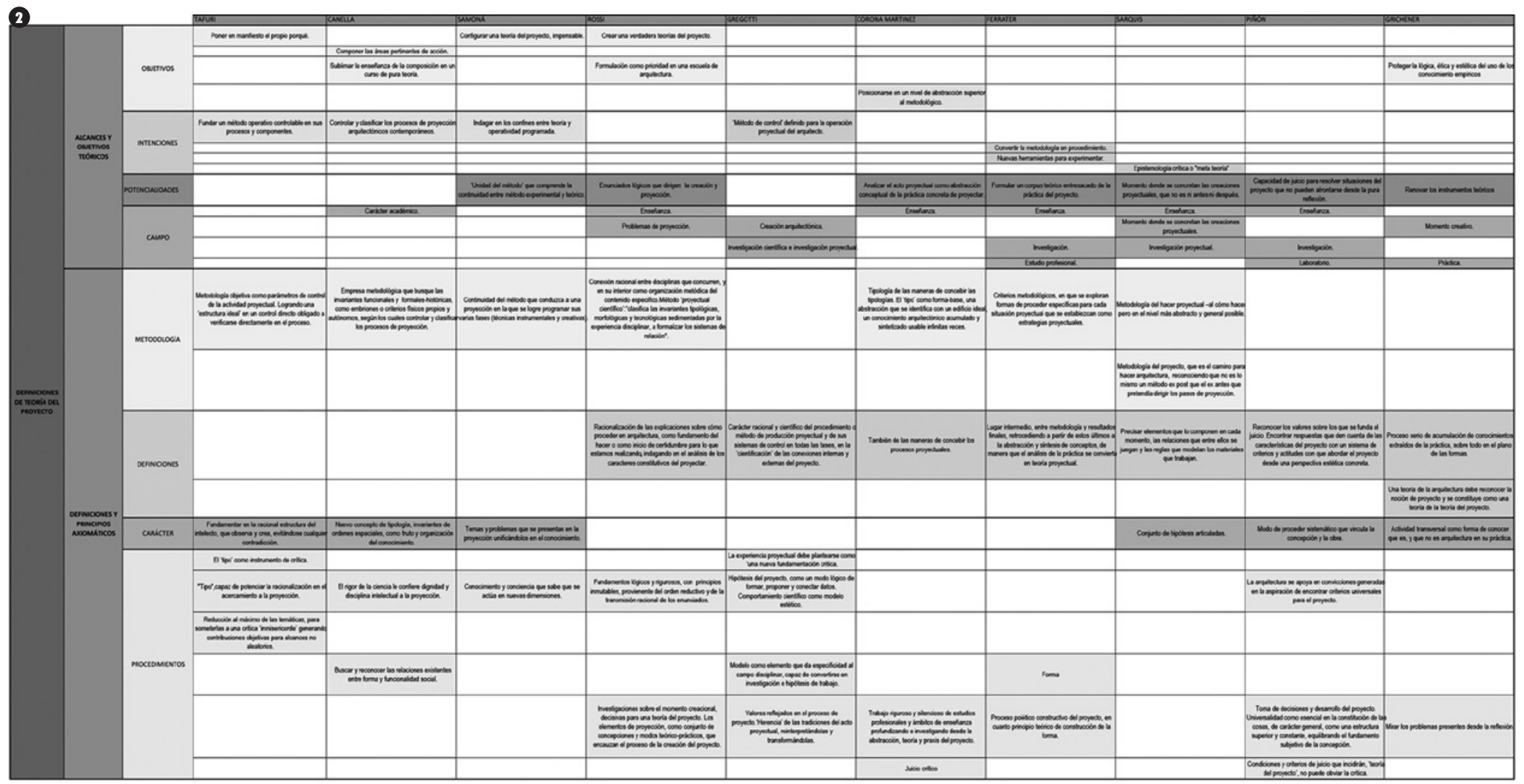

rodear el objeto de estudio por todos sus lados para arribar a la elaboración de una definición lo más completa posible en cada caso. Para la posterior contrastación de las diferentes ideas-contenidos extraídas de cada autor se confeccionaron ocho tablas comparativas por veintiún temáticas conceptuales afines surgidas desde la descomposición misma de los discursos de los diversos autores.

Una vez descompuesto los discursos individuales y contrastados con los otros referentes, se procedió a detallar las diferentes posturas referidas a la problemática, buscando sus relaciones internas y externas, enfatizando en sus dependencias, para su integración o disyunción teórica. Posteriormente, se intentó ensayar la estructuración de un discurso globalizante, que trate de organizar un corpus lógico de contenidos.

\section{EL SURGIR DE LA NECESIDAD POR CONSTRUIR TEORÍAS DEL PROYECTO}

"Porque hecha la cosa

no te pese haberla hecho,

y hayas de decir:

esto no quisiera, más quisiera aquello".

L. B. AlbertI.

En el año 1966 el IUA de Venecia convocó a jóvenes arquitectos para dictar conferencias sobre la posibilidad de generar Teorías del Proyecto, estas conferencias fueron recopiladas en el libro TEORíA DE LA PROYECTACIÓN ARQUITECTÓNICA (Canella, et al. 1968), en esas presentaciones coinciden autores como Rossi, Gregotti o Tafuri y son ellos quienes presentan los primeros argumentos sistemáticos a favor de generar Teorías del Proyecto.
Aldo Rossi en UNA ARQUITECTURA PARA LOS MUSEOS (1968), comienza reconociendo que no existen, o son muy escasas, las Teorías del Proyecto y que su formulación debe ser prioridad en una escuela de arquitectura; pues constituye su objetivo específico, teniendo innegable preferencia sobre otras investigaciones y representando "el momento más importante, básico, de toda arquitectura" (1968, p.

185). En paralelo a eso elabora su crítica al estado de la arquitectura de esos años; consistente en cuestionar enfáticamente la invención y aplicación de teorías tomadas de otras disciplinas (como economía, sicología y lingüística) para pretender encontrar un enunciado que sirva de explicación y sea aplicable al hecho arquitectónico, una especie de salvavidas argumental, tan usual aún en nuestros días. 
En esta línea es que Linazasoro (1984) nos dice que resulta preciso recuperar una auténtica Teoría de la Proyectación, la que debe estar formulada, con referencias a una teoría de la arquitectura, es decir a una teoría general del hecho arquitectónico que no debe ser confundida con una específica Teoría del Proyecto, pues "Esta última tiene un carácter más restringido en abierta relación con la praxis" (1984, p. 19).

Por su parte, Seguí (1996) nos recalca que parece una atrocidad que dentro de las escuelas no se produjeran Teorías del Proyecto, siendo que dedican la mitad de su tiempo y también sus mejores recursos humanos al aprendizaje de proyectos; sin embargo quienes más profundizan en la necesidad de constitución de Teoría del Proyecto son los miembros del grupo de investigación de crítica arquitectónica (Arkrit) de la Escuela de Madrid (ETSAM), ellos afirman que:

"La Teoría del Proyecto es simplemente escasa e irrelevante y mucho más compleja que la simple yuxtaposición o combinatoria de la tratadística, la historia y otras ciencias de la arquitectura y aunque no ha podido ocupar, más que en una mínima parte del territorio que le corresponde dentro de la teoría de la arquitectura, ella pasa ineludiblemente por la crítica, pues cierto rigor científico constituye su instrumento poético ideal" (Miranda, et al. 2004, p. 1)

Conclusivamente es Samonà (1968) quien, en el convencimiento que las investigaciones serán infructuosas y faltas de precisión para afrontar los problemas que se nos presentan a través de la búsqueda de la misma proyectación, reconoce la falta endémica de una Teoría de la Proyectación que, como refrenda el grupo Arkrit, debe ser sólida y científica, desligándose radicalmente del gusto y la moda, para avanzar en depurar las buenas obras de arquitectura y así consolidar la necesaria capacidad de juicio crítico, pues "la médula de una teoría del proyecto es su misión de obtener leyes generales por medio de las estructuras comunes a diferentes obras relacionadas por estructuras geométricas abstractas, las cuales a su vez habrán sido extraídas de la ciencias propias e internas de la arquitectura" (Miranda, 1999, p. 101).

\section{HACIA UN DISCURSO CONSTRUCTIVO: COMPONENTES DE LA TEORÍA DEL PROYECTO}

Una vez estudiados los argumentos de los autores seleccionados, es posible identificar tres niveles de aproximación, que permiten poner en comparación las ideas vertidas, logrando configurarse, de la forma más clara y aglutinante posible los argumentos que vinculan las distintas materias. A menudo es posible advertir, como quedará de manifiesto en los epígrafes siguientes, confusión teórica y redundancias en tópicos que se trasladan de un campo de reflexión a otro. Sin periuicio de ello, y siguiendo el carácter "fenoménico" e imparcial de la investigación, el estudio de los diferentes contenidos expresa el reconocimiento de caminos comunes, divergentes y en algunos casos enfrentados.

\section{ALCANCES Y OBJETIVOS TEÓRICOS}

Cada autor establece objetivos que dan sentido y cuerpo a su intención teórica, Samonà (1968) y Rossi (1968) proponen crear una verdadera Teoría del Proyecto, impensable aún -para el primero- e inexistente para el segundo, Tafuri (1968) expresa, por una parte, la intención de poner de manifiesto el propio porqué de la creación arquitectónica, junto a Gregotti (1968) y Canella (1968), intentan fundar parámetros de control definidos para la operación proyectual donde supervisar y clasificar los procesos, indagando en los confines entre teoría y operatividad (Samonà, 1968).

A favor de una renovación de los instrumentos teóricos desde la creación y práctica proyectual se encuentran autores como Corona Martínez
(1998) que advierte la necesidad de abstraer conceptualmente en un nivel superior al metodológico. Ferrater (2006) ve posible fundar un corpus teórico entresacado desde la práctica del proyecto, Sarquis (2000) se centra en el momento justo donde se concretan las creaciones proyectuales; como Samonà (1968) que busca la unidad metódica entre lo experimental o práctica y lo teórico, también Rossi (1968) que busca encontrar los enunciados lógicos que dirigen la creación y proyección protegiéndose a la lógica, la ética y la estética del uso de los conocimientos netamente empíricos (Grichener, 2007).

En una línea distinta, Piñón (1998) intenta conocer la magnitud y el sentido del cambio que propuso la modernidad en el modo de concebir la forma, declarando que desarrollar una Teoría del Proyecto es plantear aquellos fundamentos estéticos. Destacando la necesidad de adquirir capacidad de juicio para resolver situaciones de proyecto que no pueden afrontarse solo desde la reflexión. Finalmente está el objetivo de avanzar científicamente en la constitución de la Teoría; esa línea es abordada por Rossi (1968) y Gregotti (1968) cuando apuntan a la necesidad de saber de qué se ocupa esta teoría primero, y a la inserción de la estructura de investigación científica en el proceso y práctica proyectual.

La importancia de esta noción es vista como revolucionaria por Samonà (1968) y sintetizada por Canella (1968) al declararla como constituyente de un verdadero modelo de cultura, en otras palabras, la filosofía del arquitecto. Para Ferrater (2006) es el material que verdaderamente le importa al arquitecto y sirve para su quehacer proyectual, Sarquis (2000) le endosa la responsabilidad de aclarar el rol que ha de cumplir el proyecto en la arquitectura $y$, también, el actuar como nexo entre esta y la sociedad. Su posición en relación a la teoría de la arquitectura es vista por Grichener (2007) como una derivación o, como aclara Rossi, un instante de la teoría arquitectónica que se ubica entre esta y la creación proyectual. 
Estas teorías se insertan en campos de desarrollo, destacándose cuatro: la academia o enseñanza; la creación proyectual arquitectónica y los problemas de la proyección; la vinculación entre ámbitos de enseñanza y nuevas formas de práctica profesional, sea en laboratorios, centros de investigación o estudios profesionales; $y$, finalmente, la incipiente investigación proyectual.

\section{COMPONENTES TEÓRICOS COMUNES A LOS AUTORES ESTUDIADOS}

En este epígrafe serán tratadas distintas áreas de indagación por los autores. Cada una se instala como una esfera de búsqueda amplia que aglomera argumentos comunes, son activos componentes que clarifican ámbitos de incumbencia proporcionando temáticas que circunscriben el nivel de las definiciones teóricas.

\section{ORIGEN, DIAGNÓSTICO Y SENTIDO DE LA CRÍTICA}

La crítica al contexto socioeconómico y disciplinar se muestra como un comienzo invariable. Desde la ideología existe un consenso transversal sobre la difícil situación producida por el sistema capitalista en los métodos de producción arquitectónicos, donde los dueños del capital han reemplazado a los gobernantes, sumergiéndonos a una sociedad de consumo (Tafuri, 1968). Surge la arquitectura del espectáculo, como un híbrido insensato que relaciona banalidad estética y derroche económico, aspirando a ser exitosa y no de calidad (Piñón, 2006). Para Samonà (1968) la crisis de la proyectualidad se relaciona con el aumento de variables del acto creativo impulsadas por el sistema sociopolítico-empresarial, vistas por Grichener (2007) como una legitimación de la construcción del mundo en base a oportunidades de negocios.

Se reconocen ciertas consecuencias de esta condición, donde Tafuri y Piñón coinciden en reconocer la consumibilidad y fragilidad de un proceso que deja de considerarse como obra, para ser visto como producto o ganancia. En esta crisis semántica del lenguaje, advertida por Tafuri, y respaldada por Sarquis y Piñón, se detecta que la arquitectura se mueve en un figurativismo experimental, utilizando restos de modernidad, haciendo un juego frívolo y hedonista de formas sin sentido, confiándose todo en la imagen, entrando peligrosamente en la dependencia de la coyuntura y la expresión personal. También hay cuestionamientos (Canella y Rossi, 1968) a la inclusión de metáforas tomadas desde otras disciplinas para pretender fundar enunciados y explicaciones al hecho proyectual. Aflora la visión negativa sobre apoyarse en la subjetividad, que fomenta la arbitrariedad y permite incluso, para Rossi, que el carácter ambiguo ascienda a sistema, potenciando la idea de que esto pudo ser de otra manera (Gregotti, 1968).

Profundizando, Sarquis advierte que el profesional se encuentra limitado al uso de formas sin sentido, coincidente con Samonà (1968) que cuestiona la tendencia a convertir cualquier instrumento de proyección en un método general. Tafuri (1968) apunta a la pérdida de valor cognoscitivo de la disciplina al destruirse el sentido de forma y quebrarse la relación entre signo y significado, cosa que Piñón (2006) considera fundamental, pues con la pérdida de la reflexión se perpetúa el acrecentamiento de la separación entre idea y razón, polos que están unidos en la síntesis creadora del proyecto.

\section{PROYECCIÓN: ACTO Y PROCESO}

La proyección es entendida como un momento cognoscitivo y el instante donde más nos acercamos a una teoría (Rossi, 1968), una especie de invocación a un objeto para hacerlo existir, específico de la modernidad arquitectónica, que se presenta como hegemónico para llegar a la creación. Sarquis (2003) la reconoce como una actividad creativa, productiva y poética, complementada por Piñón (2006) al verla como el modo específico de entender la forma, manejando diversas disciplinas autónomas en un sistema de sistemas.

El proceso de proyección se apoya en elementos decisionales, una especie de sucesión de actos encadenados, propositivos y reflexivos que intentan concebir un objeto dotado de estructura definida con criterios de forma consistente (Piñón, 2006). Donde se dan diversos juicios y momentos de comprensión e invención contraponiéndose el momento crítico y el sintético; estos momentos implican un rehacerse continuo que le confieren -según Gregotti (1968)- un orden líquido y según Ferrater (2006) un orden en espiral, para permitir someter constantemente a revisión todo el proceso.

Si Tafuri (1971) apoya el carácter racional del proceso, Gregotti (1968) se muestra decidido a insertar el modelo científico como instrumento de control para su estructuración; lo que deriva en la aparición de la metodología, que será defendida en su propuesta de encontrar una método de carácter objetivo. Para Sarquis (2003) la metodología es un procedimiento o conjunto de operaciones para obtener un fin; en cambio para Ferrater (2006) es un mecanismo de control o blindaje por el cual entregar un carácter hermético al proceso, evitando las intrusiones externas no deseadas.

La coincidencia absoluta de los autores en el poder productor de conocimiento del acto proyectual, se nutre de entender el proceso como una operación crítica, donde por medio de la razón y la invención se desarrolla la capacidad de juicio, que puede pensarse como una valoración sobre el hacer mismo, o como criterio para definir la forma que, en cuanto principio generatriz, se muestra sin existencia real constituyéndose como una proyección sobre la realidad del objeto, esto deriva en que sea entendida como la materia y el objeto de la proyección. Piñón (2006), aún mostrándose en contra de valorar el proceso, al entenderlo como una falta de capacidad de juicio, con la que se intenta validar el resultado final, avanza más 
que todos en profundizar sobre el juicio estético y la capacidad de desarrollarlo, para el juicio es la actividad básica del proyectar permitiendo el reconocimiento de la dimensión formal, donde se busca la calidad que, como condición sintética, se refiere a la totalidad del producto.

\section{RELACIÓN ENTRE TEORÍA Y PRÁCTICA}

Para varios autores la relación entre teoría y práctica es diluida, al punto de no entenderse la separación entre un antes y un después o entre pensar y proyectar arquitectura. De ese modo la línea entre creador y lo creado se borra y el nexo entre crítica y proyecto, reflexión y acción -en definitiva- teoría y práctica se interpreta ausente. Sarquis (2003) asevera que el momento proyectual posee reglas que se renuevan, cuestionan y reformulan la dimensión teórica, incluso como teoría de la práctica; desde ese punto de vista -esa dimensión- se hace inexistente, mostrándose indisolublemente ligada a la práctica, por eso dirá que no existen tareas carentes de teoría, y menos en relación pacífica con su práctica (Sarquis, 2000). Grichener (2007) destaca la actitud dialógica que reconoce entre ambos conceptos, de hecho para Ferrater (2006) la teoría proyectual está en el análisis de la práctica y para Piñón (2006) la teoría está en los proyectos y no en los textos.

Para Gricehener (2007), surge la necesidad de abrir la teoría a la práctica del proyecto, de la enseñanza y de la actividad profesional; apoyado en el convencimiento que desde la teoría surgen buenas preguntas que orientan y nutren estudios empíricos y estos, a su vez, refinan o desechan preguntas teóricas. Este conocimiento proyectual, de carácter transmisible, hace a Corona Martínez (1998) refrendar el dictado teórico como un deber del taller de proyectos. Por su parte, Piñón (2006) define la teoría como un intento por encontrar, mediante la reflexión con carácter crítico, explicación a cuestiones que no la tienen aplicando el sentido común, sin ser posible reducirla a un manual de instrucciones.

\section{RELACIÓN CON EL CONOCIMIENTO Y SU GENERACIÓN}

Esta categoría, indisolublemente ligada al punto anterior, por la condición del proyecto de ser objeto de conocimiento que se produce en el acto mismo de su creación, como instante donde se pierde la dualidad teoría-práctica, es vista como la capacidad del proyectar de generar conocimiento activo producido en la experimentación y la acción, provocando que ambos términos -conocimiento y acción- sean complementarios e integrados entre sí, logrando una identificación radical (Tafuri, 1968). Si Rossi (1968) concluye que es posible entender este conocimiento al modo de ciencia; Gregotti (1972) va más allá y postula la implementación de sistemas racionales de valoración proyectual, pues - a su juicio- la ciencia se propone al mundo artístico, como modo de indagar y conocer.

Bajo el convencimiento colectivo que la disciplina elabora principios y se transmite según ciertas leyes, aparece la necesidad de poder orientar conscientemente esos procesos y conocimientos; para ello se invoca la necesidad de desarrollar el lenguaje o discurso crítico, proponiéndose incluso una epistemología crítica; en el convencimiento que el carácter crítico puede actuar sobre el hacer, volviendo el proceso proyectual un objeto de reflexión en, o desde, la acción. Cobra importancia el discurso lingüístico, que para Sarquis (2007) y Piñón (2006), es el único que permite reflexionar sobre el mismo, posicionándose como constitutivo de la disciplina, debiendo aparecer textos que correspondan a la explicitación de los principios y posiciones que enmarcan la toma de decisiones del momento creativo existente entre la concepción y la obra.

\section{INVESTIGACIÓN PROYECTUAL Y ENSEÑANZA}

Es posible detectar cierto modus operandi experimental propuesto por los autores, intentando configurar un campo de investigación -que se reconoce ausente- en torno al proyecto, pero más específicamente en torno al momento de la creación proyectual. Aquí Sarquis (2008) advierte sobre una deficiencia importante en torno a la temática: la dificultad de no contar con normas básicas consensuadas para definir la calidad de una investigación en este campo; además del desafío de colocar a la arquitectura en paridad y competencia con otros campos del saber. En este sentido Gregotti (1968) coloca la relación entre investigación científica e investigación proyectual como el problema superior donde se inserta la Teoría del Proyecto.

El campo de acción de la investigación proyectual advierte tres escalas: transversalmente se aloja dentro de los problemas genéricos que plantea la práctica del proyecto, centrándose en la experimentación creativa del momento proyectual, como procedimiento configurador de la forma y productor de conocimientos, para profundizar en sus tácticas, instrumentos, criterios y valores que permitan resolver problemas de la acción y práctica proyectual. Produciéndose una renovación de las bases experimentales y teóricas, con capacidad de enriquecer los conocimientos disciplinares para la producción arquitectural, implicando la documentación de los procesos como el análisis de los modos de hacer que, en base a determinadas teorías, metodologías y técnicas, sean configuradores de formas de reconocida calidad.

Ferrater, Sarquis y Piñón declaran el lazo entre centros de investigación o laboratorios de proyectos con la enseñanza, así se establecen entornos de experimentación formal, donde poder desarrollar, profundizar y resguardar los avances y futuros conocimientos en torno al proyecto. En este sentido Ferrater (2007) postula el vínculo entre despacho profesional y ámbito académico, permitiéndose la transmisión de conocimiento de modo reversible, cosa que no es compartida por Sarquis y Piñón quienes, desde el medio profesional, absorben problemáticas -que aisladas del contextoproducen mejoras y avances, para una renovación de los saberes. 
3. Tabla Definiciones de la Teoría del Proyecto.

4. Tabla Carácter de la Teoría del Proyecto.

3

\begin{tabular}{|c|c|}
\hline ROSSI & $\begin{array}{l}\text { Una racionalización de las explicaciones sobre cómo proceder en la proyección, } \\
\text { considerándolo como un fundamento del hacer o un inicio de certidumbre para aquello que } \\
\text { estamos realizando, indagándose en los caracteres constitutivos del proyectar. } \\
\text { Donde los elementos de proyección actúan como un conjunto de concepciones teórico- } \\
\text { prácticas que encauzan y dirigen por completo el proceso de creación. }\end{array}$ \\
\hline GREGOTTI & $\begin{array}{l}\text { Búsqueda de un carácter racional y cientifico del procedimiento o método de producción } \\
\text { proyectual y sus sistemas de control en todas las fases, proponiendo la 'cientificación' de las } \\
\text { conexiones internas y externas del proyecto. }\end{array}$ \\
\hline $\begin{array}{l}\text { CORONA } \\
\text { MARTÍNEZ }\end{array}$ & $\begin{array}{l}\text { Abstracción conceptual de la práctica de proyectar y de las maneras de concebir los procesos } \\
\text { proyectuales buscando los valores que alli se reflejan; interesándose en los modelos de } \\
\text { actuación, procesos, instrumentos y operaciones que se desarrollan. }\end{array}$ \\
\hline FERRATER & $\begin{array}{l}\text { Buscar en un lugar intermedio entre los resultados finales y la metodologia, donde por medio } \\
\text { de la abstracción y síntesis conceptual en la exploración de formas de proceder, el análisis de } \\
\text { la práctica se convierta en teoria proyectual. }\end{array}$ \\
\hline SARQUIS & $\begin{array}{l}\text { Una metodología del hacer proyectual, orientada al cómo hacer, pero en un nivel abstracto } \\
\text { superior y lo más general posible. } \\
\text { Analizar el proceso poítico constructivo del proyecto y su resultado para construir un conjunto } \\
\text { de hipótesis articuladas que funden un principio teórico de la construcción de la forma. }\end{array}$ \\
\hline PIÑÓN & $\begin{array}{l}\text { Por medio del reconocimiento de los valores sobre los que se funda el juicio, en cuanto } \\
\text { momento inevitable del proyecto, encontrar un conjunto de respuestas que den cuenta de las } \\
\text { caracteristicas peculiares del proyecto, sin que puedan reducirse a la acción de un sistema } \\
\text { concreto. } \\
\text { Un conjunto coherente de criterios para abordar los problemas que presenta la concepción y la } \\
\text { prefiguración de la arquitectura, buscando un modo de proceder sistemático que vincula la } \\
\text { concepción y la obra. }\end{array}$ \\
\hline GRICHENER & $\begin{array}{l}\text { Un proceso serio de acumulación de conocimientos extraídos de la práctica sobre todo en el } \\
\text { ámbito de las formas. }\end{array}$ \\
\hline
\end{tabular}

4

\begin{tabular}{|l|l|}
\hline TAFURI & o \\
\hline CANELLA & C \\
\hline SAMONÁ & A \\
\hline ROSSI & ris \\
\hline GREGOTTI & C \\
\hline FERRATER & T \\
\hline SARQUIS & F \\
\hline PINÓN & iñ \\
\hline GRICHENER & ' \\
\hline
\end{tabular}

Potenciar la racionalización en la proyección. Fun (1) Usar el rigor de la ciencia para conferir dignidad, disciplina intelectual y organizar los frutos de Apoyado en la razón tratar los problemas de la proyección, buscando unificarlos en el Conocimiento, teniendo conciencia que se actúa en nuevas dimensiones del saber rigurosos, aquellos principios inmutables.
Carácter cientifico -como modelo estético- para las fases de acumulación, análisis, elección, utilización, lectura de resultados y previsión de los objetivos.
Trabajo investigativo riguroso y silencioso, desarrollándose el juicio crítico personal.

Fundacional de una metodología que permita generar conocimiento permanente y reconocible desde la creación proyectual Apoyarse en la capacidad, condiciones y criterios universales del juicio estético y crítico, que incidirán en la toma de decisiones, pues el proyecto se apoya en convicciones y no en verdades.
Reflexión critica como en ciencias y tecnologia para crear un proceso serio de acumulación de
Aceptado el taller de proyectos como el lugar más específico de la formación, donde la práctica proyectual es valorada como su núcleo, se advierte entonces el sentido implícito que adquieren los métodos utilizados, lo que potencia que el aprendizaje y conocimiento se den en el hacer mismo. Mientras Sarquis (2003) advierte que las decisiones de enseñanza competen a la Teoría del Proyecto, Canella (1968) ve posible sublimar la enseñanza proyectual hacia un curso netamente teórico, donde vuelve a ser el centro el momento creativo o inventivo que, con capacidad de generar conocimiento, se muestra de gran utilidad didáctica.

\section{DEFINICIONES O PRINCIPIOS DE LA TEORÍA DEL PROYECTO}

Habiéndose visto las precisiones de objetivos, de intenciones, la importancia o potencialidades de esta teoría y sus campos de desarrollo, avanzamos a tratar sus axiomas fundamentales, que ayudarán a comprender las definiciones de cada autor, como los matices existentes incluso en las máximas medulares de sus propuestas. Veremos primeramente el llamado a abstraer y racionalizar las formas de proceder o el cómo hacer proyectual.
Bajo el consenso de definir la Teoría del Proyecto como un llamado a elaborar un conjunto de valores, explicaciones, hipótesis o criterios capaces de ser analizados, abstraídos, racionalizados y articulados desde el proceso poético de creación y la práctica proyectual, se busca el nivel de abstracción más alto y general posible para establecer un fundamento del hacer, que traiga certidumbres y se oriente al cómo hacer, abordando los problemas que presentan la concepción y prefiguración arquitectónica, de ese modo se pretende llegar a generar un conjunto de respuestas, en un proceso serio de acumulación de conocimientos, que se constituya como un principio teórico de la creación y construcción de la forma.

En la búsqueda de un modo de proceder sistémico, que vincule la concepción y la obra, los autores llaman a indagar en los caracteres constitutivos del proyectar o en los valores sobre los que se basa el juicio, buscando un conjunto de concepciones teórico-prácticas que encaucen y dirijan el proceso poético de proyección.

En el carácter de esta búsqueda por elaborar y acumular el conocimiento, existen tres aristas principales: el llamado a apoyarse en el orden científico (confiriéndole dignidad y disciplina intelectual), el acento en la racionalización intelectual y el desarrollo del juicio estético crítico que facilita la toma de decisiones. Los autores son proclives a un trabajo basado en la racionalización de los problemas de la proyección, que fundándose en el intelecto y la razón, busquen evitar la contradicción y proponer fundamentos lógicos y rigurosos.

Como consecuencia de lo anterior asoma con importancia el problema metodológico donde se distingue un transversal llamado a configurar o construir una metodología, desde dos visiones distintas, una más abstracta-teórica y la otra de corte más metódica aplicable. En este segundo enfoque se advierte la búsqueda de una objetividad que -reconociendo parámetros, tipologías y criterios- busque programar, clasificar y controlar los procesos de proyección; elaborándose, lo que Tafuri (1968) llama, una estructura ideal que verifique y controle directamente la actividad proyectual. En el otro lado de la visión metodológica, más abstracta y teórica, el llamado es a buscar los enunciados o principios ideales en el hacer mismo, reconociendo aquellos valores que dirigen la creación y proyección, detectándose los elementos atemporales y permanentes, como conjunto de operaciones o procedimientos generales, con capacidad de ser acumulables y sintetizables, en un solo discurso, convirtiéndose en estrategias proyectuales. 
5. Tabla Metodología en la Teoría del Proyecto.

6. Tabla Procedimientos e instrumentos para la elaboración teórica del proyecto.

(5)

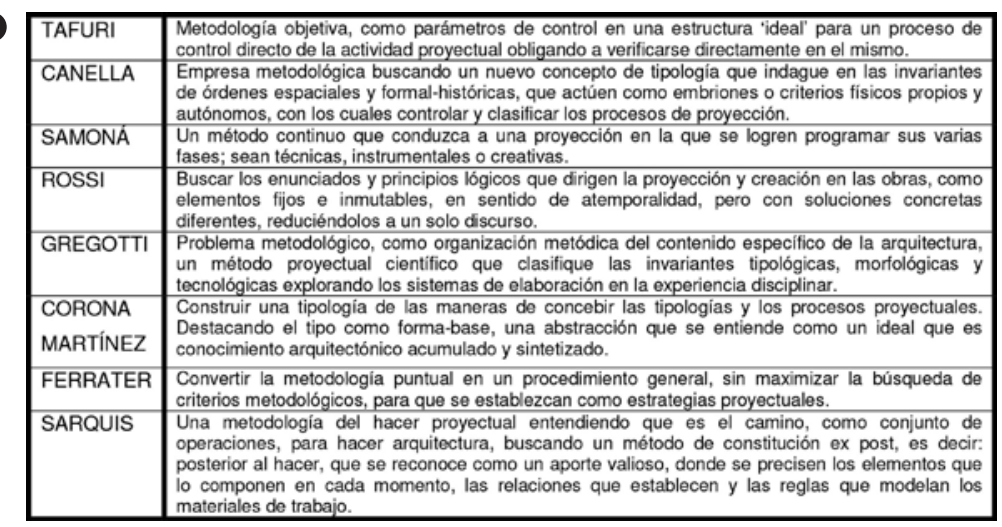

6

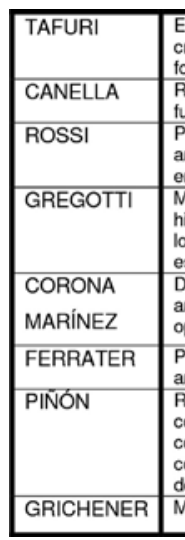
El 'tipo' como instrumento de crítica. Reducir al máximo las temáticas para someterlas a una rma como potencia generadora para avanzar hacia una estructura ideal de proyeción Rigurosa comprensión del ambiente físico buscando reconocer las relaciones entre forma y luncionalidad social. Preguntarse ¿Cómo he hecho que sean mías mis arquitecturas? Buscando en la creación arquitectural e intencionalidad los fundamentos teóricos de sus proyectos, donde la forma tendida como generatriz corresponde por derecho propio a la teoria del proyecto. irar la expeniencia proyectual como una nueva lundamentacion critica, principalmente, de la hotesis de proyecto que debe aparecer como un modo lógico de formar, proponer y conectar especificidad al campo disciplinar convirtiéndose en investigación e hipótesis de trabajo Destacando el tipo como forma-base, se entiende como un ideal que es conocimiento arquitectónico. Interesándose en los modelos de actuación, procesos, instrumentos operaciones que se desarrollan.

Profundizar desde la abstracción teórica y la praxis del proyecto en los aspectos sintácticos de la arquitectura. Reconociendo la universalidad entendida como lo esencial en la constitución de las cosas, una configuración formal general que trasciende la singularidad del problema puntual y se muestra cono una estructura superior y y constante equemplos del propio hacer pues allibrando están los criterios más auténticos surgidos desde la propia experiencia. Centrarse en la forma y los modos de construirla.
Finalmente aparece el nivel de los procedimientos o directrices declaradas para lograr configurar los principios teóricos antes expuestos. Se reconoce la necesidad de alcanzar contribuciones objetivas para producir avances no aleatorios, reduciendo al máximo las temáticas (Tafuri, 1968). También está el usar la propia experiencia y creación proyectual como materia de indagación para encontrar los criterios, las intenciones y fundamentos teóricos de sus proyectos, con una fundamentación crítica, indagando -según Gregotti- en la hipótesis del proyecto, revelándose el modo lógico como se conectan las decisiones, propuestas y formas del proyecto.

El elemento que vertebra estos procedimientos de búsqueda es la forma, entendida como potencia generatriz, por medio del estudio crítico, dialéctico y sintético de los modos de ser construida. Esto podría permitir arribar a conocimiento arquitectónico universal, entendido como lo esencial en la constitución de las cosas, una configuración formal general que se muestra como superior y constante, convirtiéndose en teoría.

A la luz de lo anterior se puede precisar que la Teoría del Proyecto busca los principios universales y atemporales que salen desde la poiesis y práctica proyectual misma, para convertirse en estrategias proyectuales ex post, que sean aplicables en la creación, prefiguración y práctica arquitectónica, transformándose más específicamente en una teoría de, para y desde el momento de la creación proyectual.

\section{UNA SÍNTESIS CONSTRUCTIVA A MODO DE CONCLUSIÓN}

Este estudio respeta en todo momento la neutralidad y distancia necesaria del método fenomenológico utilizado para intentar precisar el contenido del objeto de estudio. En ningún caso se ha modificado la información y los contenidos extraídos de los argumentos de los autores. Ahora bien, y a la luz de los resultados, es posible arriesgar una vía para intentar producir un hilo constructivo provisional, que permita profundizar y avanzar, en base a lo recopilado y sistematizado, en un resultado provechoso para la disciplina y la docencia.

Si bien es prematuro y requiere de mucho más estudio el esbozo de un modelo definitivo de innovación teórica, se ve viable advertir ciertos lineamientos y ámbitos definibles para establecer claridad en torno a lo investigado, conteniendo un desafío paradójico: cerrar la investigación abriéndola, con la intención de que este estudio no funcione como cierre, y se transforme en prólogo. Esta síntesis que intenta ser concluyente de lo investigado, aporéticamente pretende abrir nuevos horizontes regidos por la búsqueda de la unidad entre teoría y práctica proyectual.
Para lograr esta síntesis constructiva se trabajó en base a las definiciones logradas anteriormente, se construyeron dos modelos: uno apuntando a situar el momento de búsqueda de los principios universales y atemporales para la elaboración de un conjunto de valores, explicaciones, hipótesis o criterios capaces de ser analizados, abstraídos y racionalizados. Este modelo se denominó de "Extracción Teórica" y consistió en ubicar a cada autor (en base a sus argumentos) en un diagrama que se compone de tres conjuntos los que, unidos entre sí, representan a la creación proyectual, a la práctica proyectual y al producto proyectual terminado.

El segundo modelo, llamado de "Aplicación Teórica" repite el diagrama de conjuntos anterior, pero esta vez procede a ubicar los autores de acuerdo al momento que advierten, en sus discursos, donde se utiliza la construcción teórica anterior. Es el momento donde los elementos atemporales y permanentes-como conjunto de operaciones o procedimientos generales- con capacidad de ser acumulables y sintetizables se aplican como estrategias proyectuales, para la constitución del proyecto.

El modelo de Extracción Teórica permite precisar que si bien no de forma unánime, los momentos de extracción de los principios teóricos son mayoritaria e indistintamente la práctica y el producto proyectual. El producto es visto como 
un elemento sintético que contiene y engloba, además de ser posible desprender desde él la totalidad de lo relevante contenido en la poiesis y praxis proyectual. Es considerable que existan divergencias, como por ejemplo Jorge Sarquis, para quien la extracción se produce en la creación y la práctica, o Ferrater que centra ese proceso en la praxis, distinto a Canella que lo posiciona decididamente en el producto y particularmente importante es Rossi que ve posible extraer los principios teóricos desde cualquiera de las tres esferas potenciales.

Por su parte, el diagrama de Aplicación Teórica muestra con claridad, y exceptuando a Samonà (quien considera que los principios extraídos son aplicables solo a la práctica proyectual), la total convergencia en reconocer que los momentos de aplicación teórica son indistintamente la poiesis y praxis proyectual, como instantes donde se construye y solidifica la forma o estructura esencial interna (no figura) arquitectónica.

Esto permite definir que, de acuerdo a lo investigado y el resultado de los diagramas anteriores, la noción de Teoría del Proyecto es usada indistintamente para referirse tanto al producto (resultado u obra proyectual) como a su práctica y creación. Considerando lo anterior se definen cuatro esferas que interactúan y corresponden a la Teoría del Proyecto, ellas son: poiesis, praxis, producto (como proyecto terminado) y teoría con un sentido circular donde una da paso a la otra, sin necesariamente ser secuenciales, siendo difícil reconocer un principio y un final, en ese sentido es que se puede advertir un sentido en espiral continuo entre estos cuatro componentes.

A modo de cierre, se propone un diagrama provisional del proceso permanente de elaboración teórica del proyecto desde su poiesis, su práctica, su resultado y su teoría. Este esquema presenta un sentido circular dejando de manifiesto su secuencialidad constante, decididamente sin origen y término, en una vinculación de instancias que muestran la ligazón y reciprocidad entre teoría y práctica como partes de un proceso unitario.
7. Modelo de Extracción Teórica.

8. Modelo de Aplicación Teórica.

9. Modelo provisional del proceso continuo de elaboración teórica del proyecto.
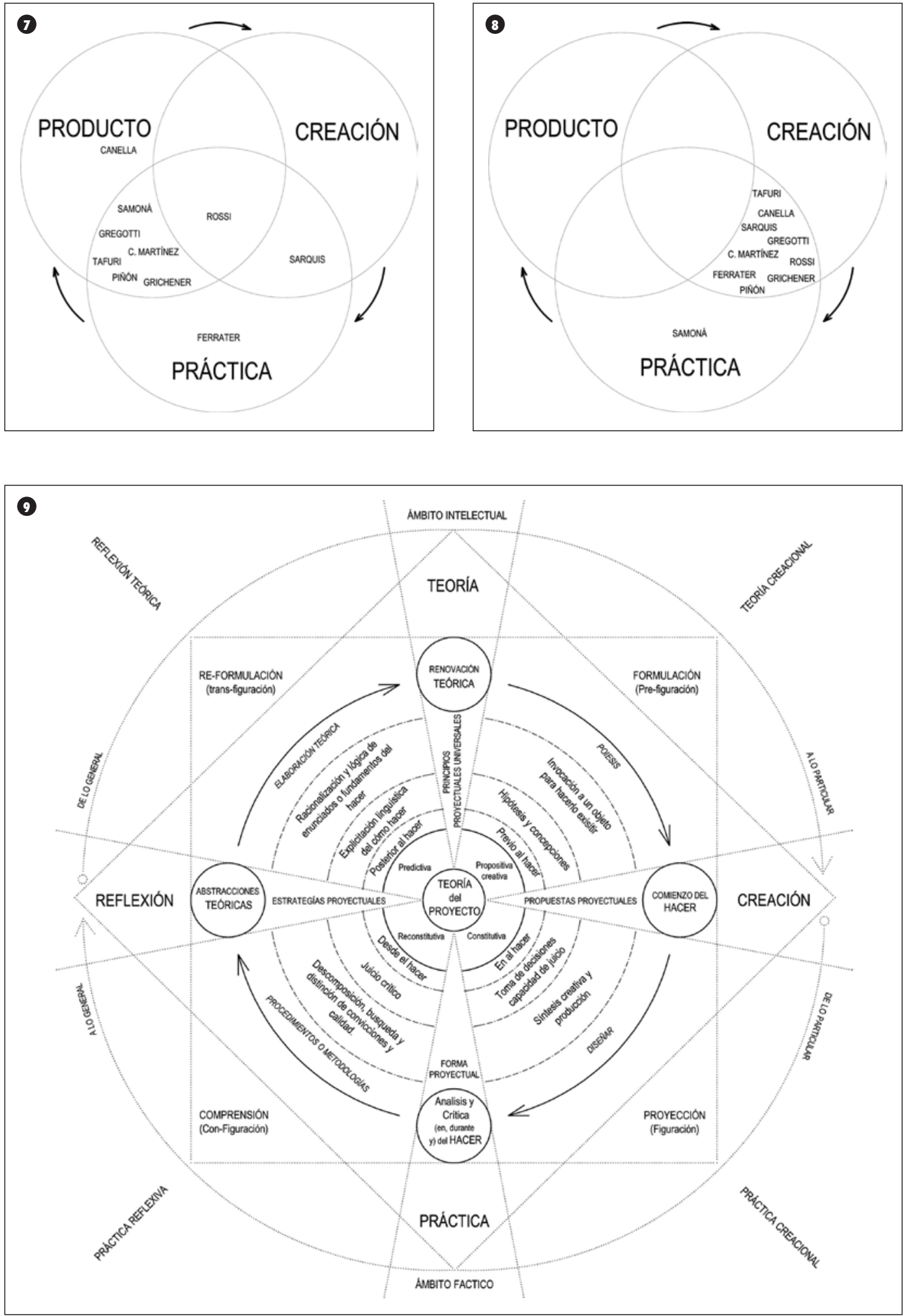


\section{REFERENCIAS}

Ábalos, I. (2005). Atlas Pintoresco (Vol. 1): El observatorio. Barcelona: Editorial Gustavo Gili.

Canella, G., Coppa, M., Gregotti, V., Rossi, A., Samonà, A., Scimemi, G., Semerani, L. y Tafuri, M. (1968). Teoría de la Proyectación Arquitectónica. Barcelona: Editorial Gustavo Gili.

Canella, G. (1968). Desde el laboratorio de la composición. En G. Canella, M. Coppa, V. Gregotti, A. Rossi, A. Samonà, G. Scimemi, L. Semerani, y M. Tafuri, Teoría de la Proyectación Arquitectónica (135-158). Barcelona: Editorial Gustavo Gili.

Corona Martínez, A. (1998). Ensayo sobre el Proyecto. Buenos Aires: Editorial Nobuko.

Delueze, G. y Guattari, F. (1997). Mil Mesetas: capitalismo y esquizofrenia. Valencia: Editorial Pre-Textos.

Eisenman, P. (1984). El fin de lo clásico. Perspecta (21), 73-153.

Ferrater, C. (2006). Sincronizar la geometría: paisaje, arquitectura y construcción. Barcelona: Editorial Actar D.

Ferry, G. (1991). El trayecto de la formación: los enseñantes entre la teoría y la práctica. Barcelona: Editorial Paidós.

Gregotti, V. (1968). Los materiales de la proyectación. En G. Canella, M. Coppa, V. Gregotti, A. Rossi, A. Samonà, G. Scimemi, L. Semerani, y M. Tafuri, Teoría de la Proyectación Arquitectónica (209240). Barcelona: Editorial Gustavo Gili.

Gregotti, V. (1972). El Territorio de la Arquitectura. Barcelona: Ediciones Gustavo Gili.

Grichener, S. (2007). Perspectivas, prácticas y teoría de la arquitectura. En J. Sarquis (Comp.), Coloquio Teoría de la Arquitectura y Teoría del Proyecto (145-152). Buenos Aires: Editorial Nobuko.

Hays, K. M. (2000). Architecture. Theory since 1968. Nueva York: Columbia Books of Architecture.

Herreros, J. (2012). Presentación. En J. GarcíaGermán, Estrategias operativas en arquitectura.
Técnicas de proyecto de Price a Koolhaas (12. 15). Buenos Aires: Editorial Nobuko

Jencks, C. (1980). El Lenguaje de la Arquitectura Posmoderna. Barcelona: Editorial Gustavo Gili. Leach, N. (1999). La an-estética de la arquitectura. Barcelona: Editorial Gustavo Gili.

Linazasoro, J.I. (1984). Apuntes para una Teoría del Proyecto. Valladolid: Secretariado de Publicaciones Universidad de Valladolid.

Mabardi, J.F. (2012). Maestría del Proyecto. Apuntes para la práctica de la enseñanza del proyecto. Concepción: Ediciones Universidad del Bío-Bío.

Marina, J.A. (2004). Crónicas de la Ultramodernidad. Barcelona: Editorial Anagrama.

Miranda, A. (1999) Ni Robot Ni Bufón. Manual para la Crítica de Arquitectura. Madrid: Ediciones Cátedra S.A.

Miranda, A. (2008) Columnas para la Resistencia. Variaciones sobre ciudad, arquitectura y subcultura. Madrid: Editorial Mairea Libros.

Miranda, A., Pina, R., Casqueiro, F., Colmenares, S. y Maruri, N. (2012). La crítica de arquitectura como modelo de investigación. Valencia: 4 IAU $4^{\mathrm{a}}$ Jornadas Internacionales sobre Investigación en Arquitectura y Urbanismo.

Montaner, J.M. (1999). Arquitectura y Crítica. Barcelona: Editorial Gustavo Gili.

Ockman, J. (2000). The Pragmatist imagination. Think About "thing in the making". Nueva York: Princeton Architectural Press.

Piñón, H. (2006). Teoría del Proyecto. Barcelona: Ediciones UPC.

Piñón, H. (1998). Curso Básico de Proyectos. Barcelona: Ediciones UPC.

Rossi, A. (1966), La Arquitectura de la Ciudad. Barcelona: Editorial Gustavo Gili.

Rossi, A. (1968). Una Arquitectura para los museos. En G. Canella, M. Coppa, V. Gregotti, A. Rossi, A. Samonà, G. Scimemi, L. Semerani, y M. Tafuri, Teoría de la Proyectación Arquitectónica (185208). Barcelona: Editorial Gustavo Gili.
Samonà, G. (1968). Introducción. En G. Canella, M. Coppa, V. Gregotti, A. Rossi, A. Samonà, G Scimemi, L. Semerani, y M. Tafuri, Teoría de la Proyectación Arquitectónica (9-20). Barcelona: Editorial Gustavo Gili.

Sarquis, J. (2000). Investigación Proyectual: historia de las teorías, los procedimientos y las técnicas. Teorías, Praxis y Poiesis. Revista Área (200), 3-25.

Sarquis, J. (2003). Itinerarios del Proyecto: ficción epistemológica Vol. 1. Buenos Aires: Editorial Nobuko.

Sarquis, J. (2007). La teoría de la arquitectura, entre la sophía y la episteme. En J. Sarquis (Comp.), Coloquio Teoría de la Arquitectura y Teoría del Proyecto (17-42). Buenos Aires: Editorial Nobuko.

Sarquis, J. (2008). Producción de Conocimiento en Arquitectura, Diseño y Urbanismo. Buenos Aires: Editorial Nobuko.

Seguí de la Riva, J. (1996). Escritos para una Introducción al Proyecto Arquitectónico. Madrid: Ediciones Departamento de ideación gráfica E.T.S. de Arquitectura de Madrid.

Sokal, A. y Bricmont, J. (1997). Imposturas intelectuales. Barcelona: Editorial Paidós.

Solà-Morales, I. (2003). Inscripciones. Barcelona: Editorial Gustavo Gili.

Solà-Morales, I. (1995). Diferencias. Topografía de la arquitectura contemporánea. Barcelona: Editoria Gustavo Gili.

Tafuri, M. (1968). Las estructuras del lenguaje en la historia de la arquitectura moderna. En G. Canella, M. Coppa, V. Gregotti, A. Rossi, A. Samonà, G. Scimemi, L. Semerani, y M. Tafuri, Teoría de la Proyectación Arquitectónica (21-50). Barcelona: Editorial Gustavo Gili.

Venturi, R. (1966). Complejidad y Contradicción en la Arquitectura. Barcelona: Editorial Gustavo Gili. 